\title{
Toroidal Equilibrium in an Iron-Core Reversed Field Pinch
}

\author{
Guthrie Miller
}

\section{DISCLAIMER}

This report was prepared as an account of work sponsored by an agency of the United States Government. Neither the United States Government nor any agency theroof, nor any of their employees, makes any warranty, express or implied, or assumes any legal liability or responsibility for the accuracy, completeness, or usefulness of any information, apparatus, product, or process disclosed, or represents that its use would not infringe privately owned rights. Reference herein to any specific commercial product, process, or service by trade name, trademark, manufacturer, or otherwise does not necessarily constitute or imply its endorsement, recommendation, or favoring by the United States Government or any agency thereof. The views and opinions of authors expressed herein do not necessarily state or reflect those of the United States Government or any agency thereof.

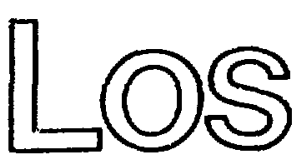


TOROIDAL EQUILIBRIUM IN AN IRON-CORE REVERSED FIELD PINCH

by

Guthrie Miller

\section{ABSTRACT}

An analytic theory of toroidal equilibrium in the ZT-4OM reversed field pinch is obtained, including effects of iron cores and resistive shell. The iron cores alter the form of the equilibrium condition and cause the equilibrium to be unstable on the shell resistive time scale.

\section{INTRODUCTION}

In this report an analytic theory of toroidal equilibrium in tie ZT-40M reversed field pinch is obtained. The primary object of this work is to elucidate the roles of iron cores and imperfectly conducting shell in ZT-40M, as these important experimental variables have not up to now been considered in reversed-field-pinch equilibrium calculations.1,2 specifi= reasons for performing these investigations are the following: 1) For long time scale experiments, the vertical field required for equilibrium needs to be supplied by external windings rather than by the shell. To design a vertical field control system, an equation of motion of the vertical field is required. Such an equation is supplied here. The proper objective of the control system will only be clear after experimental studies, but it is argued here that the objective should be the elimination of the shell equilibrium current, which causes error fields. This objective can be accomplished and is not limited by shell time constant. 2) The positional stability of the equilibrium in ZT-40M has not up to now been examined. It is found here that the iron cores destabilize both horizontal and vertical motions on a shell resistive time scale. The air-core st bilization technique of field indexing (elliptical shaping of the plasma) is useless in such a situation and feedback stabilization is necessary. 3) The in-out asymmetry of the iron in ZT-40M, 
where a larger fraction of space is occupied by iron at smal1 rather large major radius, has been proposed as a perturbing influence on the equilibrium. This effect is quantitatively calculated here. 4) The experimental measurement of the shell resistive time constant in $\mathrm{ZT}-40 \mathrm{M}$, by observing the penetration of vertical. field, cannot be interpreted properly unless the important effect of the iron cores is taken into account.

Following the well-known work of Shafranov, ${ }^{3}$ an aspect-ratio expansion is used. This method is particularly appropriate for reversed field pinches, which tend to have large aspect ratios. The theory has been derived from a somewhat different starting point than the usual. In particular, the mair toroidal constraint, a condition that the body force per unit length acting on the plasma be zero, is derived using an elementary Maxwe11 stress argument. This perhaps clarifies the physical meaning of the well-known Shafranov formula. The internal structure of the equilibrium is obtained by straightforward aspect-ratio expansion of the equilibrium equations. An interesting side issue comes to light from numerical studies of the internal structure of reversed-field-pinch equilibria, namely, that plasma pressure has very little effect on the plasma shift, as long as natural magnetic field variables are held fixed.

The principal object of study here, the iron core and shell problem, requires the calculation of external magnetic fields including shell, external windings, and iron cores. This is a complicated task, and, for reasons of space, only a general description of the method is possible. Final results are given for the specific geometrical configuration of $2 \mathrm{~T}-40 \mathrm{M}$.

\section{METHOD}

The curved cylindrical coordinate system $r, \theta, z$ is illustrated in Fig. 1. It is convenient to also refer to the following coordinate systems related to $r, \theta, z$ : the cylindrical system $R, \phi, Z$, with

$$
\begin{aligned}
& R=R_{0}-r \cos \theta \\
& Z=r \sin \theta
\end{aligned}
$$




$$
\phi=z / R_{0}
$$

the Cartesian system $X, Y, Z$, with

$$
\begin{aligned}
& X=R \cos \phi \\
& Y=R \sin \phi ;
\end{aligned}
$$

or the curved Cartesian system $x, y, z$, with

$$
\begin{aligned}
& x=r \cos \theta \\
& y=r \sin \theta
\end{aligned}
$$

The solution of the equilibrium problem will be obtained by first-order expansion in $r / R_{0}$. The zero-order, cylindrical, plasma equilibrium is specified by two profiles, given beforehand, that we take to be the parallel current $\sigma=j \cdot B / B^{2}$ and the pressure $p$. The zero-order fields are found by solving the equations

$$
\begin{aligned}
& B_{\theta}^{\prime}=\sigma B_{z}-\frac{B_{\theta}}{r}-\frac{B_{\theta}}{B^{2}} p^{\prime} \\
& B_{z}^{\prime}=-\sigma B_{\theta}-\frac{B_{z}}{B^{2}} p^{\prime}
\end{aligned}
$$

with the boundary conditon $\mathrm{B}_{\theta} \rightarrow \sigma \mathrm{rB}_{z} / 2$ as $r+0$, where denotes the derivative with respect to $r$. To first order in $r / R_{0}$, the magnetic field is changed by having additional small terms $\sim r / R_{0}$. These terms are given in the Appendix. The toroidal effect of main importance here, however, is a boundary condition that expresses the fact that the net force per unit length on the plasma is zero. The net force acting on the plasma depends on magnetic fields 
produced by windings external to the plasma, so we shall need a method for calculating the external magnetic field.

The net force per unit length can be derived by the following elementary argument. Consider the stress acting across the curved cylindrical surface 1 and end plane 2 shown in Fig. 2. The force acting in the $\mathrm{X}$ direction is

$$
\begin{aligned}
F_{X} & =\int(j \times B-\nabla p) \cdot \hat{X} d V \\
& =\int B \cdot \hat{X} B \cdot d S-\left(p+\frac{B^{2}}{2}\right) \hat{X} \cdot d S,
\end{aligned}
$$

with the surface elements given by

$$
\begin{aligned}
& \mathrm{dS}_{1}=\left(1-\frac{\mathrm{a}}{\mathrm{R}_{0}} \cos \theta\right) \mathrm{a} \mathrm{d} \theta \mathrm{d} z \hat{\mathrm{r}} \\
& \mathrm{dS}_{2}=r \mathrm{dr} \mathrm{d} \theta \hat{\mathrm{z}},
\end{aligned}
$$

and

$$
\hat{x}=(-\hat{r} \cos \theta+\hat{\theta} \sin \theta) \cos \phi-\hat{z} \sin \phi
$$

Substituting Eq. (6) into Eq. (5), we obtain

$$
\begin{aligned}
F_{X, 1} & =\int\left[\left(-B_{r} \cos \theta+B_{\theta} \sin \theta\right) \cos \phi-B_{z} \sin \phi\right] B_{r}\left(1-\frac{a}{R_{0}} \cos \theta\right) a d \theta d z \\
& +\int\left(p+\frac{B^{2}}{2}\right) \cos \theta \cos \phi\left(1-\frac{a}{R_{0}} \cos \theta\right) a d \theta d z,
\end{aligned}
$$

and 


$$
\begin{aligned}
F_{X, 2} & =\int\left[\left(-B_{r} \cos \theta+B_{\theta} \sin \theta\right) \cos \phi-B_{z} \sin \phi\right] B_{z} r d r d \theta \\
& +\int\left(p+\frac{B^{2}}{2}\right) \sin \phi r d r d \theta
\end{aligned}
$$

By taking the $\phi$ derivative at $\phi=0$ the net force per unit length is obtained:

$$
\begin{aligned}
\frac{d F_{X}}{d z} & =\int\left(-B_{r} \cos \theta+B_{\theta} \sin \theta\right) B_{r}\left(1-\frac{a}{R_{0}} \cos \theta\right) a d \theta \\
& +\int \frac{B^{2}}{2}\left(1-\frac{a}{R_{0}} \cos \theta\right) \cos \theta a d \theta \\
& +\int\left(-B_{z}^{2}+p+\frac{B^{2}}{2}\right) \frac{r}{R_{0}} d r d \theta
\end{aligned}
$$

Equation (7) is an exact result for any aspect ratio, assuming only that $p=0$ for $\mathrm{r}=\mathrm{a}$.

We now introduce an aspect-ratio expansion as follows (assuming a circular cross section as in $\mathrm{ZT}-40 \mathrm{M})$ :

$$
\begin{aligned}
& B_{r}=B_{r 1}(r) \sin \theta+\ldots \\
& B_{\theta}=B_{\theta 0}(r)+B_{\theta 1}(r) \cos \theta+\ldots \\
& B_{z}=B_{z 0}(r)+B_{z 1}(r) \cos \theta+\ldots,
\end{aligned}
$$

where the subscripts denote the order of the quantity in $r / R_{0}$. Substituting Eq. (8) into Eq. (7), we obtain to first and leading order 


$$
\begin{aligned}
\frac{d F_{X}}{d z} & =\pi a\left(B_{r l} B_{\theta}-\frac{a}{R} \frac{B^{2}}{2}+B_{\theta} B_{\theta l}+B_{z} B_{z l}\right) \\
& +\frac{1}{R} \int\left(p+\frac{B^{2}}{2}-B_{z}^{2}\right) \operatorname{rdrd} \theta,
\end{aligned}
$$

where the subscript 0 has been dropped. The final, we11-known, result is obtained by eliminating $\mathrm{B}_{z}$ and $\mathrm{B}_{\mathrm{z} 1}$ froin $\mathrm{Eq} \cdot(9)$, using the zero-order relation: $\left(p+B^{2} / 2-B_{z}^{2}\right) r=\left(2 p+B_{\theta}^{2} / 2\right) r-\left[\left(p+B^{2} / 2\right) r^{2}\right]^{\prime} / 2$ and the first-order vacuum field relation $\mathrm{B}_{\mathrm{zl}}=\mathrm{aB}_{\mathrm{z}} / \mathrm{K}_{0}$ :

$$
\frac{\mathrm{dF}_{\mathrm{X}}}{\mathrm{dz}}=\pi \mathrm{a}\left[\left(\mathrm{B}_{\mathrm{r} 1}+\mathrm{B}_{\theta 1}\right) \mathrm{B}_{\theta}-\frac{\mathrm{a}}{\mathrm{R}} \mathrm{B}_{\theta}^{2}+\frac{\mathrm{a}}{\mathrm{R}} \int\left(2 \mathrm{p}+\frac{\mathrm{B}_{\theta}^{2}}{2}\right) \frac{\mathrm{rdrd} \theta}{\pi \mathrm{a}^{2}}\right]
$$

The force in the $\mathrm{Y}$-direction can be obtained trivially by rotating in $\theta$ and taking $\mathrm{R}_{0} \rightarrow \infty$. We will rewrite $\mathrm{Eq}$. (10) in terms of the asymmetry parameter $\Lambda$ defined by Shafranov as follows:

$$
\frac{d F_{X}}{d z}=\frac{I_{p}^{2}}{4 \pi R} \Lambda+\frac{1}{2} I_{p}\left[B_{r l}(a)+B_{\theta l}(a)\right],
$$

where $I_{p}=2 \pi a B_{\theta}$ is the plasma current and

$$
\begin{aligned}
& \Lambda=\frac{L_{\theta}}{2}+\beta_{\theta}-1, \\
& L_{G}=\frac{2}{a^{2} B_{\theta}(a)^{2}} \int_{0}^{a} B_{\theta}^{2} r d r,
\end{aligned}
$$

and 


$$
B_{\theta}=\frac{4}{a^{2} B_{\theta}(a)^{2}} \int_{0}^{a} p r d r
$$

Numerical studies using experimentally reasonable reversed-field-pinch equilibrium profiles show that $L_{\theta}$ is not independent of $\beta_{\theta}$ when $B_{\theta}(a), B_{z}(a)$, and toroidal $f l u x$ are held fixed. In fact, $L_{\theta} / 2+B_{\theta}$ is found to be almost entirely independent of $\beta_{\theta}$ for $\beta_{\theta} \leqslant 0.2$. Figure 3 illustrates this point for a $\sigma$ profile of the form

$$
\begin{array}{lc}
\sigma(r)=\sigma_{0}, & 0<r \leqslant r_{1} \\
\sigma(r)=\sigma_{0}\left(r_{p}-r\right) /\left(r_{p}-r_{1}\right) & r_{1}<r \leqslant r_{p}
\end{array}
$$

and pressure profile

$$
p(r)=f \frac{B_{0}^{2}}{512}\left(\sigma_{0} r_{p}\right)^{4}\left[1-\left(r / r_{p}\right)^{4}\right]
$$

where $r_{p}$ is the plasma radius and $B_{0}$ is the on-axis magnetic field. The pressure is written in this way to show the fraction $f$ of the Suydam limiting pressure on axis. Field profiles calculated using the formulae given in the Appendix are shown for zero pressure and $f=0.2$. More familiar dimensionless pressure parameters corresponding to $f=0.2$ are the following: $($ plasma energy)/(magnetic field energy) $=0.1,2) \beta_{\theta}=0.14,3$ ) local $\beta$ on axis $=0.047$, and 4) $p(0) / B_{\theta}\left(r_{p}\right)^{2}=0.11$. For the equilibria shown in Fig. 3, the plasma radius is assumed to be limited by contact with the liner (1iner inside radius $=19.6 \mathrm{~cm}$ ), and the shell is assumed to be a flux surface [it is found that (shell radius)/(plasma radius) $=1.22$ ]. The value of $\Lambda$ (at the shell) changes very little, from $\Lambda=-0.068$ to $\Lambda=-0.065$, as $B_{\theta}$ is 
increased from 0 to 0.14 . For this change of $B_{\theta}$, the on-axis shift changes from $\xi(0)=0.086 \mathrm{r}_{\mathrm{p}}$ to $\xi(0)=0.085 \mathrm{r}_{\mathrm{p}}$, actually decreasing s11ghtly.

The quantity $\mathrm{B}_{\mathrm{rl}}+\mathrm{B}_{\theta \mathrm{l}}$ in $\mathrm{Eq} \cdot(11)$ is determined by solving the external magnetic field problem, using boundary conditions to match solutions for various radial regions outside of the plasma. Vacuum magnetic field solutions in $r, \theta, z$ coordinates are obtained as follows. The solutions of interest have no dependence on $z$, and for such functions

$$
\begin{aligned}
\nabla \times A= & -\frac{\partial A_{\phi}}{\partial Z} \hat{R}+\left(\frac{\partial A_{R}}{\partial Z}-\frac{\partial A_{Z}}{\partial \hat{R}}\right) \hat{\phi}+\frac{1}{R} \frac{\partial}{\partial R}\left(R A_{\phi}\right) \hat{z} \\
= & \frac{1}{r} \frac{\partial A_{z}}{\partial \theta} \hat{r}-\frac{\partial A_{z}}{\partial r} \hat{\theta}+\left[\frac{1}{r} \frac{\partial}{\partial r}\left(r A_{\theta}\right)-\frac{1}{r} \frac{\partial A_{r}}{\partial \theta}\right] \hat{z} \\
& +\frac{A_{z}}{R}(\cos \theta \hat{\theta}+\sin \theta \hat{r})
\end{aligned}
$$

A gauge can be chosen for which $A_{r}=0$. As $B_{2}=0$ outside of the plasma, $A_{\theta} \sim$ 1/r. From $B_{\theta 0}=I /(2 \pi r)$, where $I$ is the net toroidal current, $A_{z O}=-$ $[I /(2 \pi)] \log (r)$. The first-order correction $A_{z l}$ satisfies [from $\nabla \times(\nabla \times A)=0$ ]

$$
-\frac{1}{r} \frac{\partial}{\partial r}\left(\frac{\partial A_{z l}}{\partial r}\right)-\frac{1}{r^{2}} \frac{\partial^{2}}{\partial \theta^{2}} A_{z l}=\frac{I}{2 \pi R} \frac{\cos \theta}{r}
$$

The solutions of Eq. (13) are $A_{2 l}=[-\mathrm{Cr}-\mathrm{D} / \mathrm{r}-\mathrm{I} /(4 \pi \mathrm{R}) \mathrm{r} \log (r)] \cos \theta$, or, in terms of magnetic field,

$$
\begin{aligned}
& B_{r}=\left(T \frac{I}{4 \pi R} \log (r)+C+\frac{D}{r^{2}}\right) \sin \theta \\
& B_{\theta}=\frac{I}{2 \pi r}+\left(-\frac{I}{4 \pi R}[\log (r)-1]+C-\frac{D}{r^{2}}\right) \cos \theta,
\end{aligned}
$$


where $C$ and $D$ are arbitrary constants to be determined by boundary conditions.

It is not permissible to take $r \rightarrow \infty$ in Eq. (14), as this violates the basic ordering assumption $r \ll R_{0}$. Far away from the plasma, the solution is determined by asymptotic matching to the field far from a current loop. The solution is of the form given by Eq. (14), but with a particular cholce of the constant $C$,

$$
\begin{aligned}
& B_{r}=\left[\frac{I}{4 \pi R}\left(\log \frac{8 R}{r}-1\right)+\frac{D}{r^{2}}\right] \sin \theta \\
& B_{\theta}=\frac{I}{2 \pi r}+\left(\frac{I}{4 \pi R} \log \frac{8 R}{r}-\frac{D}{r^{2}}\right) \cos \theta,
\end{aligned}
$$

with only one arbitrary constant in this case.

III. CHARACTERIZATION OF IRON CORES AND SHELL

Figure 4 shows the positioning of the iron cores in ZT-40M. The iron is modeled assuming constant permeability $\mu(B=\mu \mathrm{H})$, with $\mu$ a large number ( 2000). The effect of the iron cores on the axisymmetric equilibrium fields is taken into account by assuming an annular region filled with material of permeability $\vec{\mu}$, where $\vec{\mu}$ is the axisymmetric average of the actual permeability,

$$
\bar{\mu}(x, y)=\int \mu(x, y, z) \frac{d z}{2 \pi R}
$$

or

$$
\bar{\mu}=\mathrm{f} \mu+1-\mathrm{f},
$$

where $f$ is the fraction of a toroidal circumference ( $2 \pi R$ ) occupied by iron. 
Figure 5 shows plots of $f(x, y)$ for ZT-40M, together with the simple analytic fit that will be used in this report,

$$
\mathrm{f} \cong 0.39 \frac{\mathrm{R}_{0}}{\mathrm{R}} \cong 0.39\left(1+\frac{\mathrm{r} \cos \theta}{\mathrm{R}_{0}}\right)
$$

implying the following functional form for $\bar{\mu}$;

$$
\bar{\mu}=\mu_{0}+\mu_{1} \frac{r \cos \theta}{R_{0}}
$$

Thus we approximate the discrete iron cores as a continuous annulus of magnetic material with higher permeability on the inside than outside in major radius.

The permeability $\vec{\mu}$ given by Eq. (19) does not alter the form of the solution for the magnetic field. From $\nabla \times[(\nabla \times A) / \bar{\mu}]=0$, we obtain instead of Eq. (13),

$$
\begin{gathered}
-\frac{1}{r} \frac{\partial}{\partial \mathbf{r}}\left(\mathbf{r} \frac{\partial A_{z 1}}{\partial \mathbf{r}}\right)-\frac{1}{\mathbf{r}^{2}} \frac{\partial^{2}}{\partial \theta^{2}} A_{z 1}=\frac{I}{2 \pi R} \frac{\cos \theta}{r}+\hat{z} \cdot \nabla \times\left(\frac{I}{2 \pi r} \hat{\theta} \frac{\mu_{1} r}{\mu_{0}^{R}} \cos \theta\right) \\
=\frac{I}{2 \pi R}\left(1+\frac{\mu_{1}}{\mu_{0}}\right) \frac{\cos \theta}{r},
\end{gathered}
$$

which differs from Eq. (13) by the substitution $I+I\left(1+\mu_{1} / \mu_{0}\right)$.

The shell in ZT-40M is 2.2-cm-thick aluminum alloy (with a nominal resistivity of $4.2 \mu \Omega \cdot \mathrm{cm}$ ) broken by toroidal and poloidal gaps. The shell affects the plasma equilibrium by carrying dipole currents (flowing on opposite directions on the small and large major radius sides of the torus). We make two simplifying assumptions: 1) that the shell current is uniform across the thickness of the she11, and 2) that the effects of the gaps can be 
ignored. In reality, the finite width of the shell causes a delay in penetration of vertical field, and the poloidal gap causes raptd initial penetration of vertical field. The two effects seem to approximately cancel.

These assumptions were tested by observing the behavior of the shell when the vertical field windings were driven. The geometry is illustrated in Fig. 6. For the case where only the vertical field winding is excited, the vertical flux inside the shell is found to satisfy the equation

$$
\left(1+\frac{a^{2}}{c^{2}}\right) \frac{\Delta a}{2 n} \frac{d x}{d t}+x=-2 \pi R \frac{a}{b}\left(1+\frac{b^{2}}{c^{2}}\right) I_{1},
$$

where $\Delta$ and $n$ are the thickness and resistivity of the shell and $I_{1}$ is the $\cos \theta$ Fourier component of the vertical field winding current, that is,

$$
I_{1}=\frac{1}{\pi} \sum_{i} I_{i} \cos \theta_{i}
$$

where $I_{i}$ is the current of the $i^{\text {th }}$ wire located at angle $\theta_{i}$.

Figure 7 shows $\chi_{1}(t)=-2 \pi R(a / b)\left[1+(b / c)^{2}\right] I_{1}(t)$ and $\chi(t)$ measured with toriodal flux loops compared with $\chi(t)$ obtained by solving Eq * (20). The good agreement in Fig. 7 validates to a certain extent the simplified models of both the shel1 and iron cores. Note that the iron cores have an important quantitative influence on the vertical field through the factor $1+(\mathrm{b} / \mathrm{c})^{2}=$ 1.68 .

\section{RESULTS}

The equilibrium relation is obtained by equating to zero the force per unit length given by Eq. (11). To determine $B_{r l}(a)+B_{\theta l}(a)$, we ..ust solve for the external field by piecing together, using boundary conditions, solutions of the form given by Eqs. (14) and (15) in the five regions $r<a, a<b$, $b<c, c<d$, and $d<r$, as shown in Fig. 8. The quantity $B_{r l}(a)+B_{\theta l}(a)$ is obtained from the expression for the field inside the shell, 


$$
\begin{aligned}
& B_{r}=\left[-\frac{I_{p}}{4 \pi R} \log \frac{r}{a}+C+\frac{\frac{I_{p} \xi}{2 \pi}-C a^{2}}{r^{2}}\right] \sin \theta \\
& B_{\theta}=\frac{I_{p}}{2 \pi r}+\left[-\frac{I_{p}}{4 \pi R}\left(\log \frac{r}{a}-1\right)+C-\frac{\frac{I_{p} \xi}{2 \pi}-C a^{2}}{r^{2}}\right] \cos \theta,
\end{aligned}
$$

where $I_{p}$ is the plasma current and $\xi$ is the outward shift of the flux surface at $r=a$ (see Fig. 8). Note that $B_{r}(a)=I_{p} \xi /\left(2 \pi a^{2}\right) \sin \theta$ and the vertical flux is given by $x=2 \pi \operatorname{Ra} \int_{0}^{\pi} B_{r}(a) \sin \theta d \theta=2 R I_{p} \xi / a$. The time derivative of $x$ is equal to the voltage ohmically developed by shell currents, that is,

$$
\frac{d x}{d t}=n \frac{I_{1 a}}{a \Delta} 4 \pi R
$$

where $I_{1 a}$ is the $\cos \theta$ Fourier component of the shell current.

A laborious algebraic calculation for the special case $\mu_{0} \rightarrow \infty$ determines $C$ in Eq. (21) and gives the following result:

$$
\begin{aligned}
& \frac{1}{2}\left[B_{r 1}(a)+B_{\theta 1}(a)\right]=\frac{I_{p}}{4 \pi R\left(1+\frac{a^{2}}{c^{2}}\right)}\left(\log \frac{b}{a}+\frac{a^{2}-b^{2}}{2 c^{2}}\right) \\
& \quad+\frac{I_{p}+I}{4 \pi R\left(1+\frac{a^{2}}{c^{2}}\right)}\left[-\frac{\mu_{1}}{\mu_{0}}+\log \frac{c}{b}+\frac{1}{2}\left(1+\frac{b^{2}}{c^{2}}\right)+\frac{2 \log \frac{d}{c}}{1-\frac{c^{2}}{d^{2}}}\left(1+\frac{\mu_{1}}{\mu_{0}}\right)\right] \\
& \quad-\frac{I_{1}}{2 b} \frac{b^{2}+c^{2}}{a^{2}+c^{2}}-\frac{I_{l a}}{2 a}+\frac{\xi}{a^{2}+c^{2}} \frac{I_{p}}{2 \pi}
\end{aligned}
$$


where $I$ is the current in the toroidal windings, and $I_{1}$ and $I_{1 a}$ are the $\cos \theta$ Fourfer components of the toroidal winding current and the shell current. Substituting for $I_{1 a}$ in terms of $d x / d t$ using $E q .(22)$, the equilibrium relation can be written as

$$
\begin{aligned}
& \left(1+\frac{a^{2}}{c^{2}}\right) \frac{\Delta a}{2 \eta} \frac{d x}{d t}-\frac{a^{2}}{c^{2}} x=-2 \pi R \frac{a}{b}\left(1+\frac{b^{2}}{c^{2}}\right) I_{1} \\
& \quad+a I_{p}\left[\Lambda\left(1+\frac{a^{2}}{c^{2}}\right)+\log \frac{b}{a}+\frac{a^{2}-b^{2}}{2 c^{2}}\right] \\
& +a\left(I+I_{p}\right)\left[-\frac{\mu_{1}}{\mu_{0}}+\log _{b} \frac{c}{2}\left(1+\frac{b^{2}}{c^{2}}\right)+\frac{2 \log \frac{d}{c}}{1-\frac{c^{2}}{d^{2}}}\left(1+\frac{\mu_{1}}{\mu_{0}}\right)\right],
\end{aligned}
$$

which is our final result. For convenience, quantities appearing in Eq. (24) will be redefined here: $\chi$ is the vertical flax through the shell; $\Delta$ and $n$ are the thickness and resistivity of the hell; $a, b, c, d$, and $R$ are geometrical quantities shown in Fig. 8; $I_{p}$ and $I$ are the toroidal currents in the plasma and toroidal windings; $\mu_{0}$ and $\mu_{1}$ represent the permeability of the iron and are defined by Eq. (19); $\Lambda$ is defined by Eq. (11); and $I_{1}$ is the $\cos \theta$ Fourier component the current in the vertical field winding defined by Eq. (20). Nominal values: of the parameters for ZT-40M are given in Table I (p. 17).

The result given by Eq. (23) was checked by numerically solving the Iinear system of boundary-condition equations. This numerical solution also allowed checks with tha air-core result

$$
\frac{1}{2}\left[B_{r 1}(a)+B_{\theta i}(a)\right]=\frac{I_{p}}{4 \pi R} \log \frac{b}{a}+\frac{I_{p}+I}{4 \pi R}\left(\log \frac{8 R}{b}-\frac{1}{2}\right)-\frac{I_{1}}{2 b}-\frac{I_{1 a}}{2 a},
$$

in the two limiting cases 1) $\mu_{0} \rightarrow 1, \mu_{1} \rightarrow 0$, and 2) $c \pm d$. Note that Eq. (23), which is for the case $\mu \rightarrow \infty$, is not correct in the $c \rightarrow d$ limit. The 
Iimit: $c, d+/^{\infty}$ cannot be used for checks since this is not a physical case, because of the toroidal nature of the problem.

Another check of Eq. (24) is the case with no toroidal current windings, so that $y=I_{1}=0$. In this case the equilibrium position with no shell current $(\mathrm{d} \chi / \mathrm{dt}=0)$ is shifted inward an amount $\xi=\mathrm{ax} /(2 \mathrm{RI} p)$ given by

$$
\xi=-\frac{a^{2}}{2 R}\left[\left(\Lambda+\frac{1}{2}\right)\left(1+\frac{c^{2}}{a^{2}}\right)+\log \frac{c}{a}+\frac{2 \log \frac{d}{c}}{1-\frac{c^{2}}{d^{2}}}\right.
$$

$$
\left.+\frac{\mu_{1}}{\mu_{0}}\left(\frac{2 \log \frac{d}{c}}{1-\frac{c^{2}}{d^{2}}}-1\right)\right]
$$

This result agrees with Eq. (78) of Ref. 4, except for the $\mu_{1} / \mu_{0}$ term, which should be zero for the comparison. Note that the iron asymmetry given by the $\mu_{1} / \mu_{0}$, term increases the inward shift as one would expect [behaving similarly to the $\operatorname{lcg}(d / c)$ term].

The flux equation in the air-core case that corresponds to Eq. (24) is given by

$$
\begin{aligned}
\frac{\Delta a}{2 n} \frac{d x}{d t} & =-2 \pi R \frac{a}{b} I_{1} \\
& +a I_{p}\left[\Lambda+\log \frac{b}{a}\right] \\
& +a\left(I_{p}+I\right)\left[\log \frac{8 R}{b}-\frac{1}{2}\right]
\end{aligned}
$$


V. DISCUSSION

The preceding calculations depend on several approximations that are not necessarily extremely accurate. The large aspect-ratio approximation, although valid in the plasma region, probably tends to break down in the region of the iron cores and outside. The characterization of a ferromagnetic material by a cinstant $\mu$ is questionable, but, as the actual value of $\mu$ drops out of the result for high $\mu$, the aproximation would seem to be justified in this case. Spatial constancy of $\mu$ within the iron is also assumed, which would not be true near saturation as the iron preferentiaıly saturates on the inside. Finally, the $\bar{\mu}$ characterization of the discrete iron ccres is no more than : simple, physically reasonable ansatz. It is expected, nevertheless, that the qualitative features of the problem are described correctly.

The vertical field necessary for plasma equilibrium can be supplied in two ways, as shown in Eqs. (24) and (26), directly by the vertical field windings (the $I_{l}$ term) or by the shell (the $d x / d t$ term).' The required vertical field depends on the plasma current $I_{p}$, the toroidal winding current $I$, and the plasma through the $\Lambda$ term. The volt-seconds driving the discharge are given by

$$
\int V_{z} d t=\mu_{0} R\left(I_{p}+I\right) \log \frac{d}{c}
$$

for the iron-core case and

$$
\int V_{z} d t=\left(I_{p}+I\right) R\left(\log \frac{8 R}{b}-2\right)+I_{p} R \log \frac{b}{a}
$$

in the air-core case, so $I_{p}+I$ would be smaller for iron core than for air core. This is the practical advantage of the iron cores, that the discharge can be driven with a smaller current I. The observed volt-seconds in $\mathrm{ZT}-40 \mathrm{M}$ identify $\mu_{0}$ in $\mathrm{Eq} \cdot(27)$ to be about 1000 . 
The important qualitative difference between the Iron-core and air-core cases is the $x$ term in Eq. (24), which implies that the iron-core equilibrium is unstable. Without a shell $(n \rightarrow \infty)$ this instability is rapid and would occur on an Alfven time scale. The alr-core equilibrium is neutrally stable for small displacements from Eq. (26); however, it is fairly easily shown that for large displacements ( $a<\xi<R_{0}$ ), the equilibrium is stabilized by toroidal effects (a physical analogy is a current-carrying wire ${ }^{i} r_{i}$ a uniform vertical field). The nonuniformity of $\vec{\mu}$ caused by a greater concentration of iron on the inside of the torus results in an inward bias of the equilibrium [the $\mu_{1} / \mu_{0}$ term in Eq. (24)] related to the basic cause of the instability, which is the attraction between the plasma and the iron. The iron-core instability is the same for vertical or horizontal plasma shifts, so fielo indexing does not make sense in the iron-core case.

To minimize error fields, the shell equilibrium current should be made as small as possible. This is mainly because of the poloidal gap, which severely perturbs the shell current. Also, nonuniformity of the shell (in ZT-40M the shell consists of boliced-together segmfnts) or openings in the shell would create error fields with a shell current. Zero shell current is obtained by adjustment of $I_{1}$ to give $\mathrm{dx} / \mathrm{dt}=0$ in $\mathrm{Eq} \cdot(24)$ or (26). In the iron-core case, because of the instability, a feedback system controlling $I_{1}$ is required, but in the air-core case, a programmed adjustment would be sufficient. Although from the error field standpoint, the cbjective of the vertical field control system is the elimination of shell equilibrium currents, other objectives, such as "proper" positioning of the plasma flux surfaces, can be stated. Experiments are needed to decide which objective is of greatest importance.

\section{ACKNOWLEDGMENTS}

The author is indebted to the other members of the $2 T-40$ equilibrium study team, in particular R. Moses and R. Massey, who originated some of the ideas presented in this report. He would also like to thank $M$. Bevir for pointing out several errors in the original version of the manuscript. 


\section{REFERENCES}

1. H. A. B. Bodin and A. A. Newton, Nucl. Fusion 20, 1255 (1980).

2. Don A. Baker, Lawry W. Mann, and Kurt F. Schoenberg, Nuc1. Fusion 23, 380 (1983).

3. V. D. Shaf ranov, Nucl. Fusion $\underline{3}, 183$ (1963).

4. V. S. Mukhovatov and V. D. 'Shaf ranov, Nuc1. Fusion 11, 605 (1971).

\section{TABLE I}

PARAMETER VALUES FOR ZT-40M

$$
\begin{aligned}
& R_{0}=114.3 \mathrm{~cm} \\
& a=23.1 \mathrm{~cm}, \Delta=2.2 \mathrm{~cm}, \eta_{\text {eff }}=9.6 \mu \Omega \cdot \mathrm{cm} \\
& b=28 \mathrm{~cm} \\
& c=34 \mathrm{~cm}, \bar{\mu}=1000\left(1+\frac{r \cos \theta}{R}\right) \\
& d=60 \mathrm{~cm}
\end{aligned}
$$




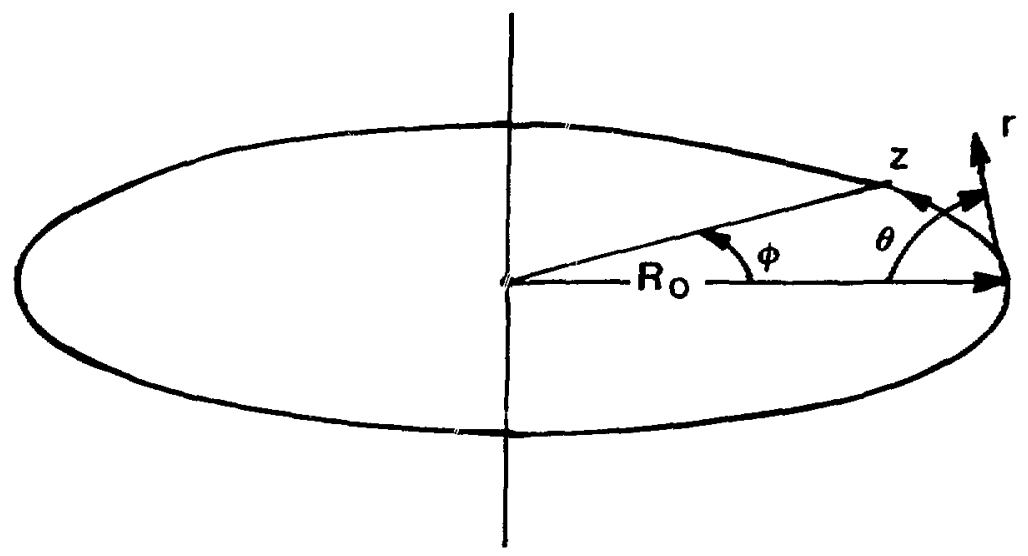

Fig. 1. Coordinate systems. The principal coordirate system used is the toroidally curved cylindrical system $r, \theta, z$.

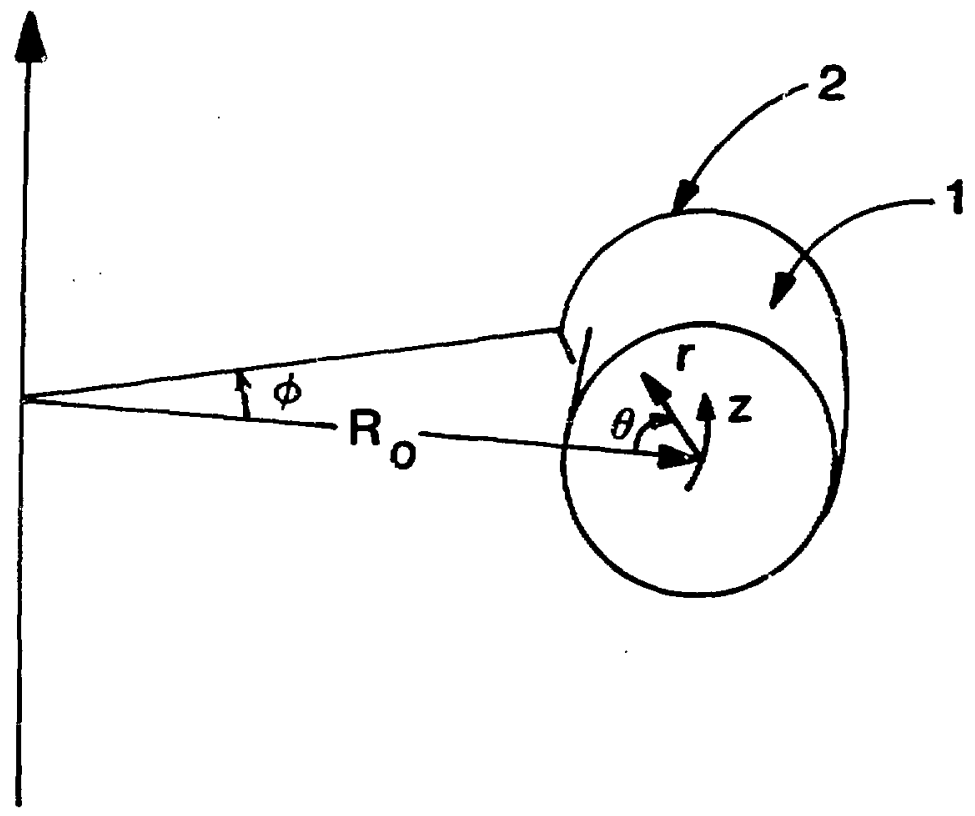

Fig. 2. Volume for which the net force acting on the plasma is calculated. 


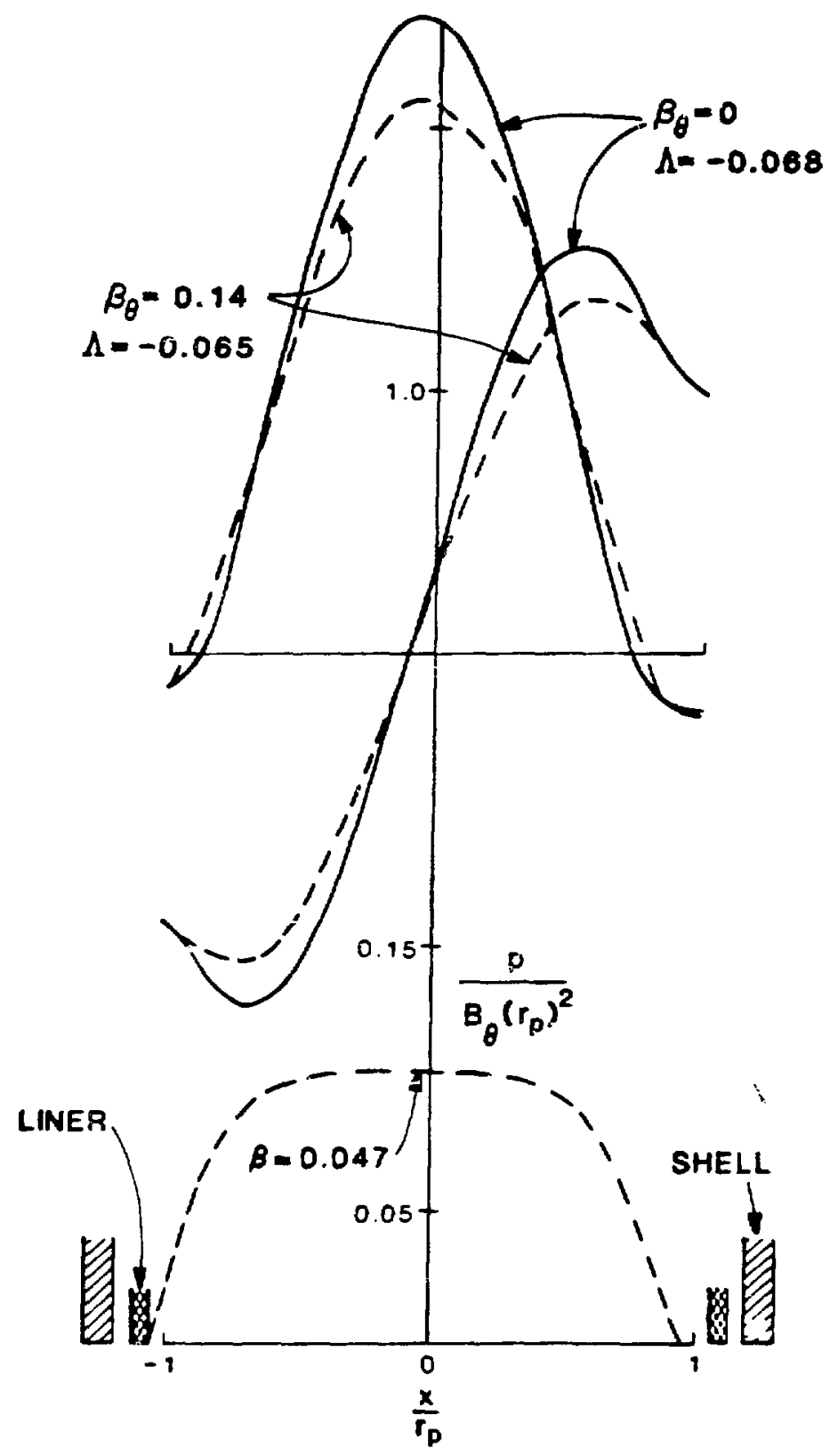

5. 3. Calculated equilibria for $B_{\theta}=0$ (solid curves) and $B_{\theta}=0.14$ (dashed curves). The upper part of the Eigure shows $B_{y}$ and $B_{z}$ at $y=0$ as a function of $x$. The plasma shift and the $B_{\theta}$ asymmetry at the shell, $\Lambda$, are nearly independent of plasma pressure. 


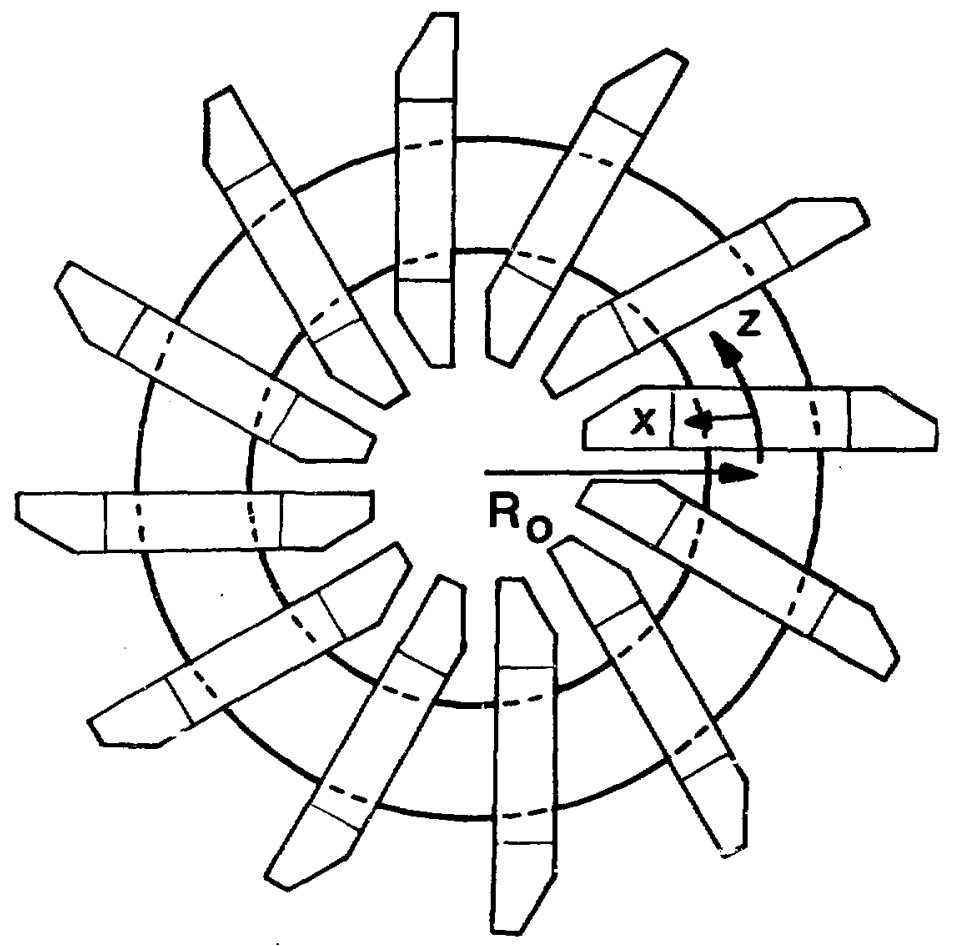

Fig. 4. Placement of Iron cores in ZT-40M.

20 


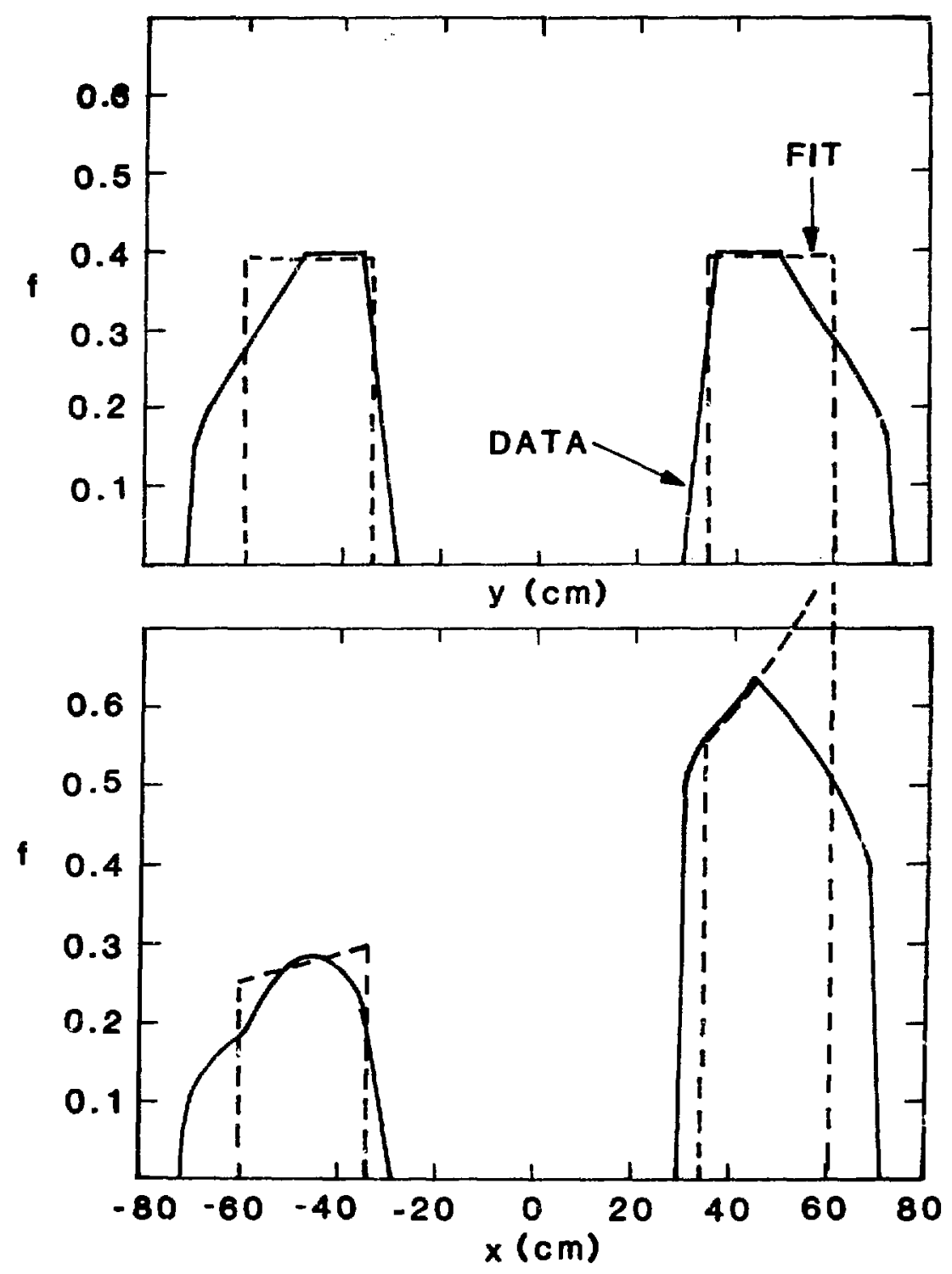

Fig. 5. Fraction of toroldal circumference occupied by iron. The coordinate $x$ is defined in Fig. 4, and $y$ is a vertical coordinate. 


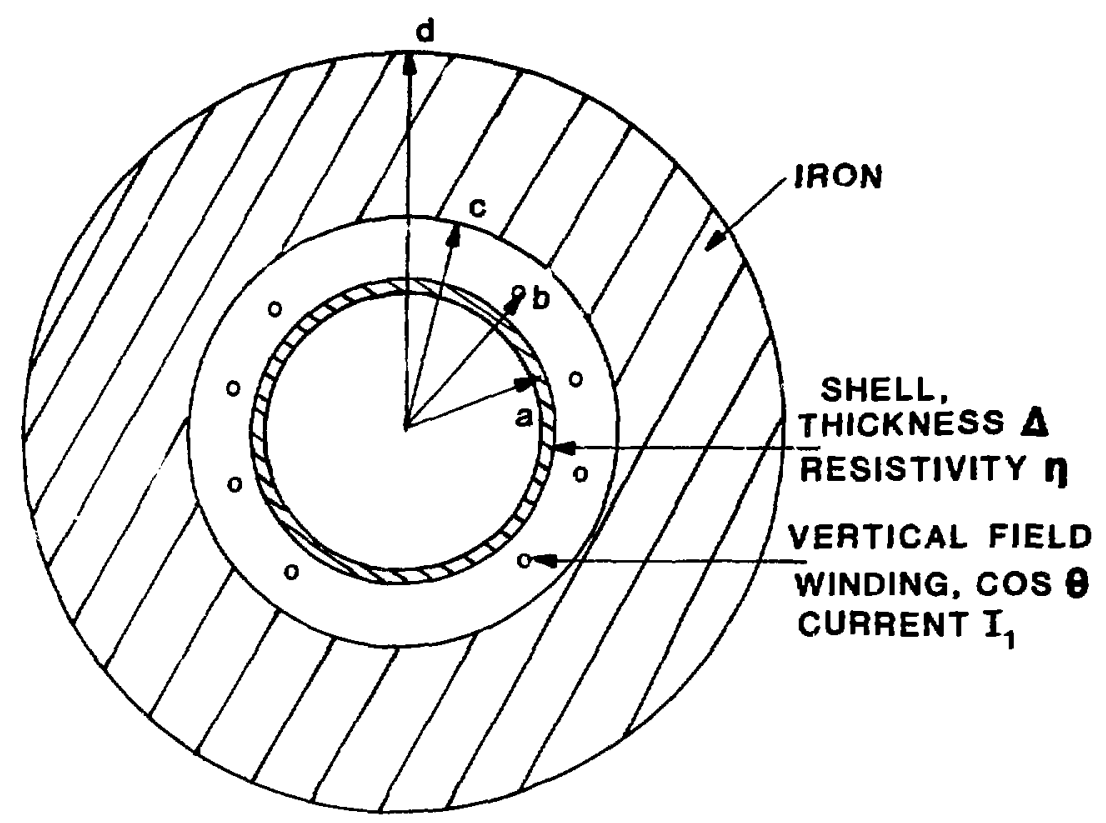

Fig. 6. Vertical field system on $\mathrm{ZT}-40 \mathrm{M}$.

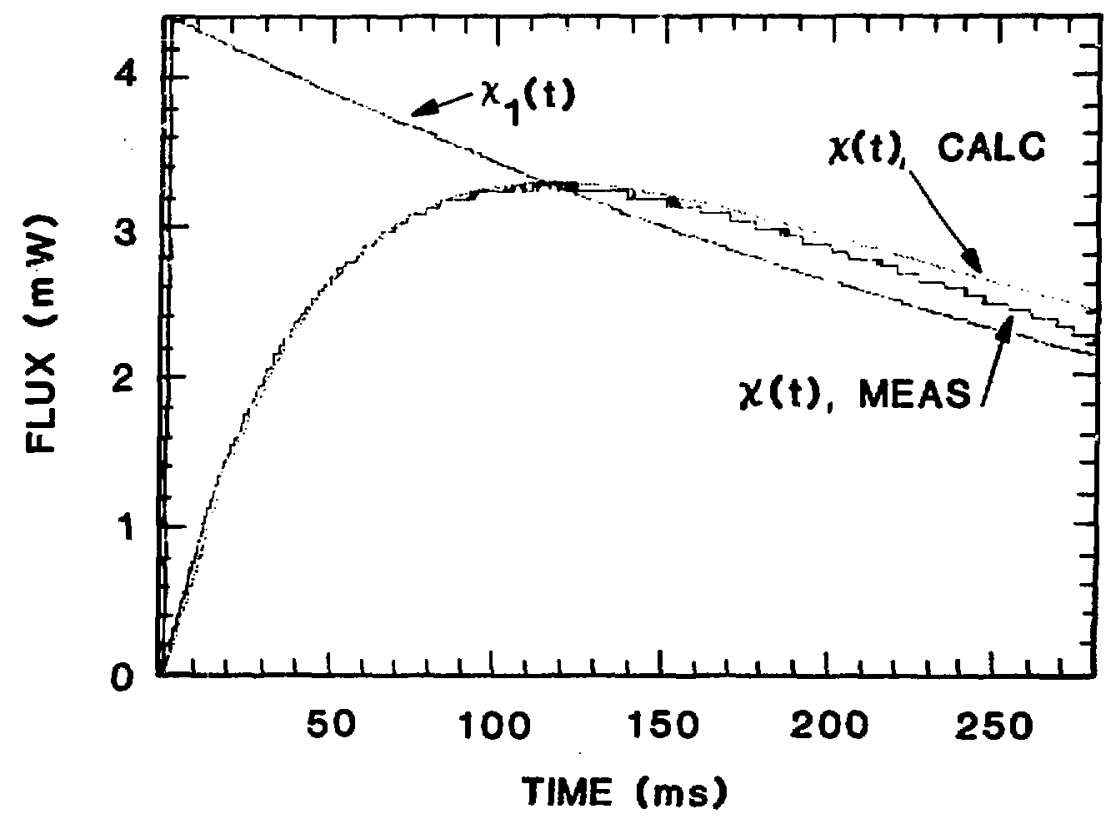

Fig. 7. Observed penetration of vertical field through the $\mathrm{ZT}-40 \mathrm{M}$ shell. 


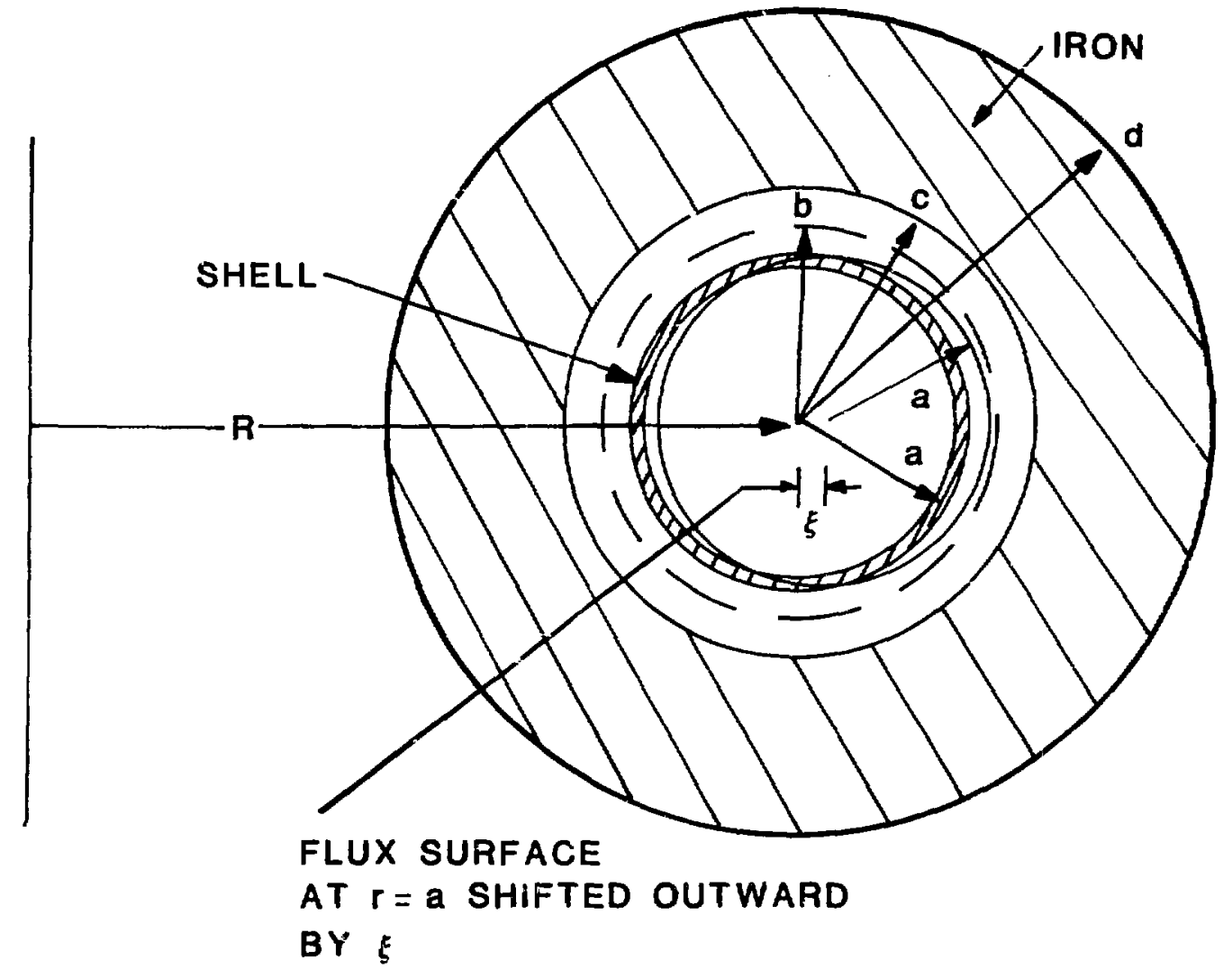

Fig. 3. Definitions of geometrical quantities. 


\section{APPENDIX - INTERNAL STRUCTURE OF THE EQUILIBRIUM}

For completeness, the formulae for the toroidal corrections to the internal equilibrium quantities are given here. The magnetic field, parallel current, and pressure are represented as follows:

$$
\begin{aligned}
& B_{r}=B_{r I}(r) \sin \theta \\
& B_{\theta}=B_{\theta 0}(r)+B_{\theta I}(r) \cos \theta \\
& B_{z}=B_{z 0}(r)+B_{z l}(r) \cos \theta \\
& \sigma=\sigma_{0}(r)+\sigma_{1}(r) \cos \theta \\
& p=p_{0}(r)+p_{1}(r) \cos \theta
\end{aligned}
$$

There are thus five unknown functions of $r: B_{r 1}, B_{\theta 1}, B_{z 1}, \sigma_{1}$, and $p_{1}$ to be determined from the five equilibrium equations, which are

$$
\begin{aligned}
& \nabla \cdot B=0 \\
& B \cdot \nabla p=0 \\
& \nabla \times B=\sigma B+B \times \nabla_{p} / B^{2}
\end{aligned}
$$

The substitution of Eqs. (A-1) into Eqs. (A-2), including the toroidal corrections to the operator $\nabla$, is straightforward but laborious. The naturaI variable is the (outward) displacement $\xi=r_{r l} / B_{\theta 0}$, which allows the resulting differential equations to be integrated. The solution is as follows:

$$
\mathrm{B}_{\mathrm{r} 1}=\frac{\mathrm{B}_{\theta} \xi}{\mathrm{r}}
$$




$$
\begin{aligned}
& B_{\theta 1}=\left(r B_{r 1}\right)^{\prime}+\frac{r B_{\theta}}{R} \\
& B_{z 1}=-\sigma r B_{r 1}-\frac{B_{z}}{B^{2}} p_{1}+\frac{r B_{z}}{R} \\
& P_{1}=\frac{r B_{r 1} p^{\prime}}{B_{\theta}} \\
& \sigma_{1}=\frac{r B_{r 1} \sigma^{\prime}}{B_{\theta}}+\frac{2 p^{\prime} B_{z}}{B^{4} B_{\theta}}\left[p_{1}+B_{z 1} B_{z}+\left(B_{\theta 1}+B_{r 1}\right) B_{\theta}\right],
\end{aligned}
$$

where the subscript 0 has been dropped. The displacement $\xi$ is given by

$$
\xi(r)=\xi(0)-\int_{0}^{r} \frac{d r_{1}}{R r_{1} B_{\theta}^{2}}\left[\int_{0}^{r}{ }^{r}\left(B_{\theta}^{2}-2 r_{2} p^{\prime}\right) r_{2} d r_{2}+C\right],
$$

where $C$ is constant. When $r=0$ is the magnetic axis of the zero-order equilibrium fleld, $c$ must be zero in order to have $\xi^{\prime}(0)$ finite. The expression for $B_{\theta 1}(a)$, where $a$ is outside the plasma, gives the following when Eq. $(A-4)$ is substituted for $\xi$ :

$$
0=\frac{I_{p}^{2}}{4 \pi R} \Lambda+\frac{1}{2} I_{p}\left[B_{r l}(a)+B_{r l}(a)\right]+\frac{\pi C}{R},
$$

which also implies that $C=0$, by comparing with Eq. (11).

A special case of Eq. $(A-4)$ is a vacuum region extending from radius $r_{p}$ to radius a. In this case

$$
\xi(a)-\xi\left(r_{p}\right)=-\frac{a^{2}}{2 R}\left[\left(\Lambda\left(r_{p}\right)+1 / 2\right)\left(1-\frac{r_{p}^{2}}{a^{2}}\right)+\log \left(a / r_{p}\right)\right]
$$

